International Journal of Modern Physics E (C) World Scientific Publishing Company

\title{
Fission of super-heavy nuclei explored with Skyrme forces
}

\author{
N. Schindzielorz, J. Erler, P. Klüpfel, P.-G. Reinhard \\ Institut für Theoretische Physik II, Universität Erlangen, D-91058, Erlangen, Germany \\ G. Hager \\ Regionales Rechenzentrum der Universität Erlangen, D-91058, Erlangen, Germany \\ Received (received date) \\ Revised (revised date)
}

\begin{abstract}
We present a large scale survey of life-times for spontaneous fission in the regime of super-heavy elements (SHE), i.e. nuclei with $Z=104-122$. This is done on the basis of the Skyrme-Hartree-Fock model. The axially symmetric fission path is computed using a quadrupole constraint. Self-consistent cranking is used for the collective masses and associated quantum corrections. The actual tunneling probability is estimated by the WKB approximation. Three typical Skyrme forces are used to explore the sensitivity of the results. Benchmarks in the regime $Z=104-108$ show an acceptable agreement. The general systematics reflects nicely the islands of shell stabilization and the crossover from $\alpha$-decay to fission for the decay chains from the region of $\mathrm{Z} / \mathrm{N}=118 / 176$..
\end{abstract}

\section{Introduction}

Self-consistent mean-field (SCMF) models have proven to be reliable tools for describing nuclear structure and dynamics, for reviews see 1|2|3. They are steadily developing to improve the quality of the predictions and to accommodate more and more observables. One of the very demanding observables are fission life-times. Fission barriers have been discussed already in the early stages of SCMF models and even been used as benchmark for calibration 4 . Fission life-times are much more involved as their computation requires not only the potential energy surface along the fission path, but also the corresponding collective masses and a safe estimate of the collective ground state correlations for the initial state. There are thus not so many self-consistent calculations of fission life-times $5 \mid 6$ - mostly using still approximate masses and quantum corrections (=zero point energies 7 ). The vast majority of calculations employs the microscopic-macroscopic method which combines shell

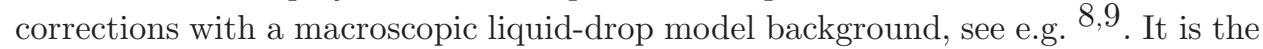
aim of this contribution to explore the systematics of fission life-times all over the landscape of super-heavy elements. This is done using the Skyrme-Hartree-Fock method as one widely used nuclear SCMF model 1 .

The paper is outlined as follows: In section 2 we briefly review the theoretical background and explain how we compute fission life-times. Results are presented 
in section 3 covering tests with experimentally known life-times, comparison with $\alpha$-decay life-times and a large scale systematics.

\section{Formal framework}

The starting point for the self-consistent microscopic description is the SHF energy functional

$$
E=E_{\text {Skyrme }}(\rho, \tau, \mathbf{J} ; \mathbf{j}, \boldsymbol{\sigma}, \boldsymbol{\tau} ; \chi)
$$

which is expressed in terms of a few local densities and currents obtained as sums over single-particle wave functions: density $\rho$, kinetic density $\tau$, spin-orbit density $\mathbf{J}$, current $\mathbf{j}$, spin density $\sigma$, and pair density $\chi$ where each occurs twice, once for protons and once for neutrons. The zero-range pairing functional is handled in a stabilized BCS ansatz to achieve smooth transitions of the occupation numbers. For details see 10. There exist various parameterizations for the Skyrme functional. In order to explore the possible sensitivity of the fission life-times to the parameterization, we confine the survey to three sufficiently different parameterizations: SkP as a force with effective nucleon mass $m^{*} / m=1$ 11, SkI3 as a fit which has very low mass $m^{*} / m=0.6$ and maps the relativistic iso-vector structure of the spin-orbit force 12, and Sly6 as a fit which has $m^{*} / m=0.7$ and includes information on isotopic trends and neutron matter 13 .

The computation of the fission path and the ingredients of the corresponding collective Hamiltonian is detailed in 14|15. We give a brief summary. The meanfield equations are derived variationally from the given energy functional. They are complemented by a quadrupole constraint to generate the fission path thus reading

$$
\begin{aligned}
& {\left[\hat{h}-\lambda \hat{Q}_{20}\right]\left|\Phi_{q}\right\rangle=\mathcal{E}\left|\Phi_{q}\right\rangle, \quad q=\left\langle\Phi_{q}\left|\hat{Q}_{20}\right| \Phi_{q}\right\rangle} \\
& \mathcal{V}(q)=E_{\text {Skyrme }}\left(\rho_{q}, \tau_{q}, \mathbf{J}_{q}, \chi_{q}\right),
\end{aligned}
$$

where $\hat{h}$ is the mean-field Hamiltonian and $\rho_{q}$ is the local density for the state $\left|\Phi_{q}\right\rangle$, similarly for $\tau_{q}, \mathbf{J}_{q}$, and $\chi_{q}$. The optimal fission path should, in fact, be generated by adiabatic time-dependent Hartree-Fock 716. The quadrupole constraint is a plausible and generally used approximation to that. To compute the collective mass along the fission path, we need to explore the dynamical response of the system to changing deformation, commonly called self-consistent cranking. To that end we use the collective-momentum operator $\hat{P}_{20}$ (and its collective momentum p) as additional constraint. The $\hat{P}_{20}$ is deduced as generator of deformation, the response to $\hat{P}_{20}$ creates a momentum-dependent path and subsequently total energy, from which the mass is finally obtained as second order term in collective momentum, altogether

$$
\begin{aligned}
& \hat{P}_{20}: \quad \hat{P}_{20}\left|\Phi_{q}\right\rangle \propto \mathrm{i} \partial_{q}\left|\Phi_{q}\right\rangle \\
& {\left[\hat{h}-\lambda \hat{Q}_{20}-\mu \hat{P}_{20}\right]\left|\Phi_{q p}\right\rangle=\mathcal{E}\left|\Phi_{q p}\right\rangle,} \\
& B_{20}=\left.\frac{1}{2} \partial_{p_{20}}^{2} E\left(\rho_{q p}, \ldots\right)\right|_{p=0}
\end{aligned}
$$


Analogously, the momentum of inertia $\Theta_{x}$ for rotations about the $x$ - and $y$-axis is computed by self-consistent cranking. The potential $\mathcal{V}$ as given in eq. (2b) has to be augmented by quantum corrections to account for angular-momentum projection and spurious zero-point quadrupole motion 7 yielding the net potential

$$
\mathcal{V} \longrightarrow V=\mathcal{V}-B_{20}\left\langle\hat{P}_{20}^{2}\right\rangle-\frac{1}{8\left\langle\hat{P}_{20}^{2}\right\rangle} \partial_{q}^{2} \mathcal{V}-\frac{\left\langle\hat{J}_{x}^{2}+\hat{J}_{y}^{2}\right\rangle}{2 \Theta_{x}}
$$

Having determined the properly corrected collective potential $V\left(\alpha_{20}\right)$ and inverse collective mass $B(q)$, we compute the fission life-time in the standard semiclassical fashion using the WKB expressions for tunneling probability $W$ and repetition time $T$ :

$$
W=\exp \left(-2 \int_{b}^{c} d q \sqrt{\frac{V(q)-E}{B_{20}}}\right) \quad, \quad T=\hbar \int_{a}^{b} d q \frac{1}{\sqrt{B_{20}(E-V(q))}} .
$$

The integrals are evaluated by trapezoidal rule with separate handling at the divergent $\sqrt{E-V}$ at the classical turning points. A quantity which enters very critically these expressions is the tunneling energy E, which is also the ground state energy of the nucleus before fission. That is computed with great care in a fully quantum-mechanical treatment of the collective quadrupole oscillations using all five quadrupole degrees-of-freedom, for details of that part of the calculations see 14[15.

It is to be noted that the fission path employs only axially symmetric shapes. One knows from actinides that triaxial shapes can produce barriers which are 0 $2 \mathrm{MeV}$ lower. The present results are thus to be understood as an upper limit on barriers and lifetimes. Actinides show the famous double-humped barrier. The outer barrier dissappears for SHE leaving only one, the formerly inner barrier 17 . This barrier is related to still symmetric shapes while asymmetry develops quickly when stepping down further towards fission. The fact that we have only one barrier simplifies the survey of fission properties of SHE.

\section{Results and discussion}

Fig. 1 shows collective potential and mass along the fission path of ${ }^{260} \mathrm{Rf}$. The quantum corrections on the potential (lower panel) reduce the barrier by an important amount making 3 to 6 orders lower life-times than would be obtained with the raw potential 7 . One recognizes a steep increase of the energy at deformation $\alpha_{20} \approx 0.6$ connected with a quick change of hexadecapole momentum $\alpha_{40}$. The system moves from one ridge (with high $\alpha_{40}$ ) to another ridge (with low $\alpha_{40}$ ). The segregation of the fission landscape into ridges is well known for actinides, see e.g. 18, still visible for that smaller SHE, and much smoothened with further increasing system size. The quadrupole mass (upper panel) shows large fluctuations. High values are related to regions of level crossings. The detailed pattern are quantitatively important and can hardly be simulated by some constant collective mass. 
Fig. 1. Example of potential energy (lower) and collective mass (upper) along the axially symmetric fission path of ${ }^{260} \mathrm{Rf}$. For the potential energy, we show the raw expectation value $\mathcal{V}$ and the corrected one $V$, see eq. (4).

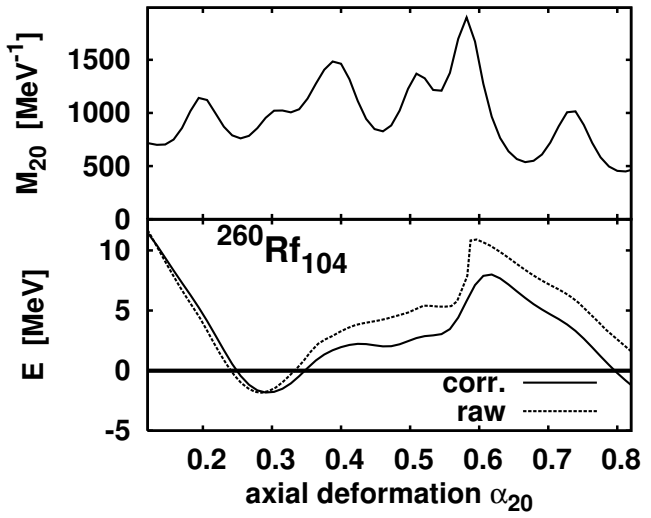

Fig. 2. Fission lifetimes computed with three different Skyrme parameterizations, as indicated, and compared with data from

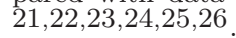

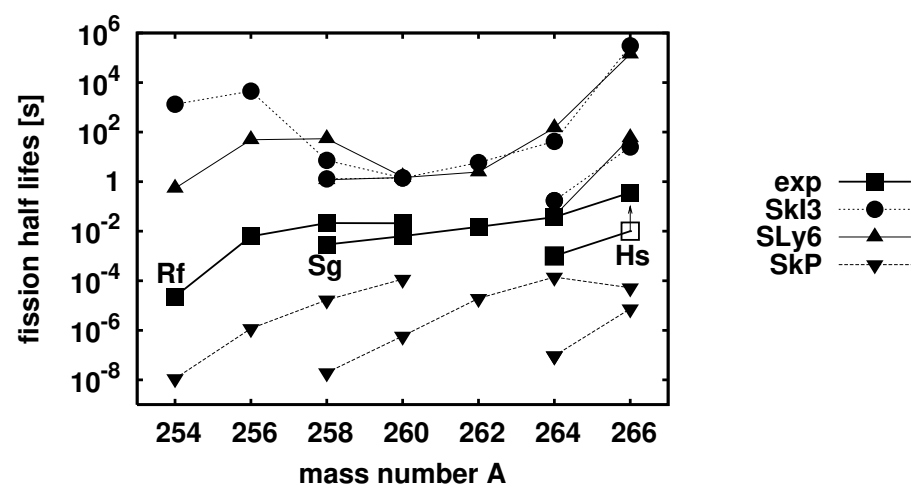

As a benchmark, we compare in fig. 2 with experimental results available at the lower edge of SHE. There are large differences in the predictions from the various forces. But the experimental values are nicely bracketed by the band of predictions and the trends within each force compare well with experiment. The deviations of \pm 4 orders of magnitude look huge. But they seem bearable in view of the extremely delicate balance in computing the tunneling rates.

We are now going to present systematics of results all over the landscape of SHE where we consider all elements which are found to be stable against immediate nucleon emission at ground state and along the whole fission path. Fig. 3 shows the fission barriers. They agree in value and systematics with the previous survey of barriers 17. One sees two islands of enhanced fission stability (high barriers), one of deformed SHE around Z/N=104/152 and the other of spherical SHE around $120 / 184$. The magic neutron number $N=184$ is also clearly marked while no unambiguous sign of a magic proton number can be found in that upper island 27. All three forces agree in predicting a ridge of very low barriers between the upper and the lower stability island. Barriers however, are mainly indications of stability. The full information on path, mass, and quantum corrections is required to calculate life-times. 


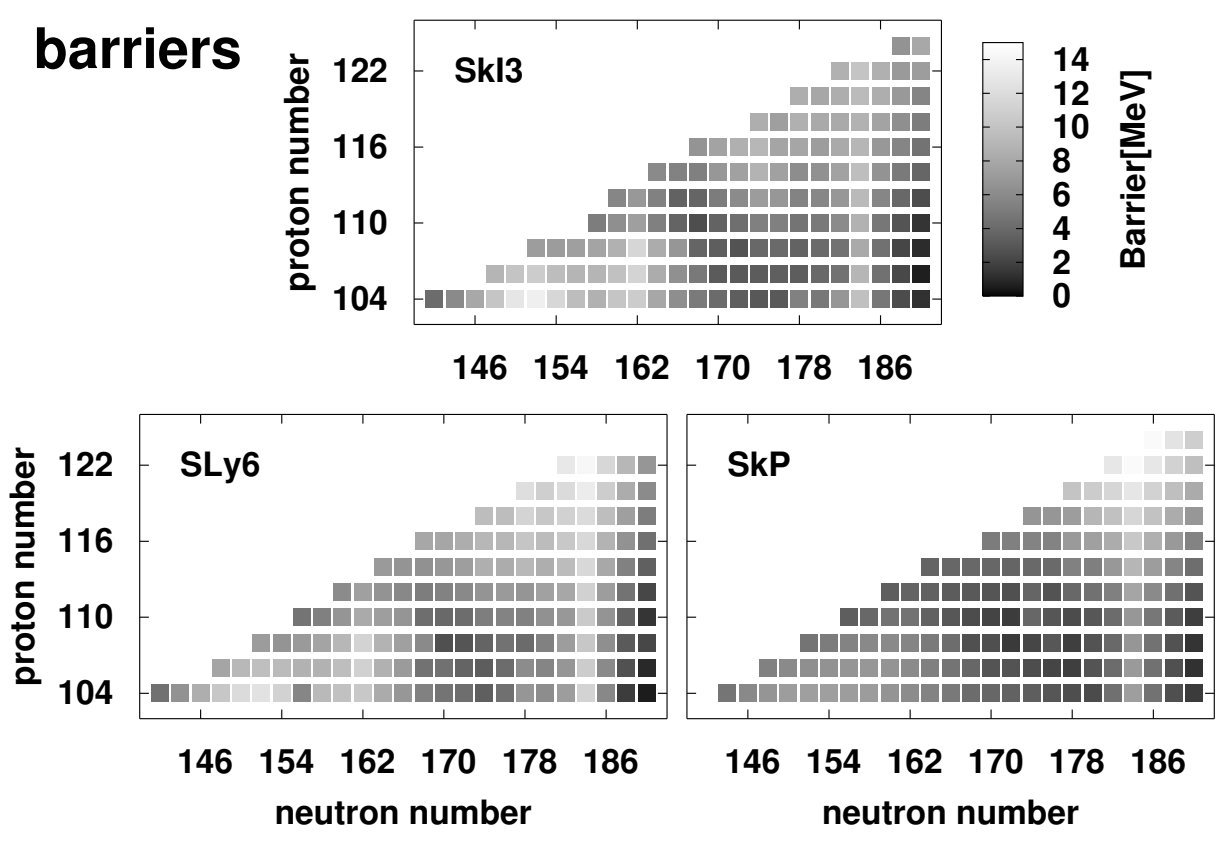

Fig. 3. Fission barriers for a broad variety of super-heavy elements and for three Skyrme parameterizations as indicated.

Fig. 4] shows the corresponding fission life-times. The difference in barrier heights from 0 to $12 \mathrm{MeV}$ translates to life-times from almost immediate decay to $10^{16} \mathrm{~s}$, demonstrating again the enormous sensitivity of fission life-times to any ingredient in its computation. The long lived SHE are found in the two islands of stability. Practically immediate decay appears in the ridge of instability between the islands. The variance of the predictions is moderate in the islands (see also fig. 22) and significantly larger in the unstable region. The latter region is not only unstable but also very hard to control theoretically.

The competing decay channel for many SHE is $\alpha$-decay. We have computed the $\alpha$ life-times from the $Q_{\alpha}$ reaction energies using a recently improved recipe 28 based on the Viola systematics. Fig. 5 shows the results. The $\alpha$ life-times change much more smoothly. There are no shell effects visible and the variance of life-times all over the landscape is very much smaller. Comparing with fig. 4, one sees that $\alpha$-decay is indeed the dominating decay channel in the islands of stability. Stepping down from the upper island, one sees a crossover of $\alpha$-decay to spontaneous fission at about $\mathrm{Z}=112$ or 110 , much in accordance with the experimental findings so far, see e.g. 19 .

The relation between $Q_{\alpha}$ value and $\alpha$ life-time is comparatively simple when adopting the Viola systematics 28 . It is interesting to ponder whether one could 


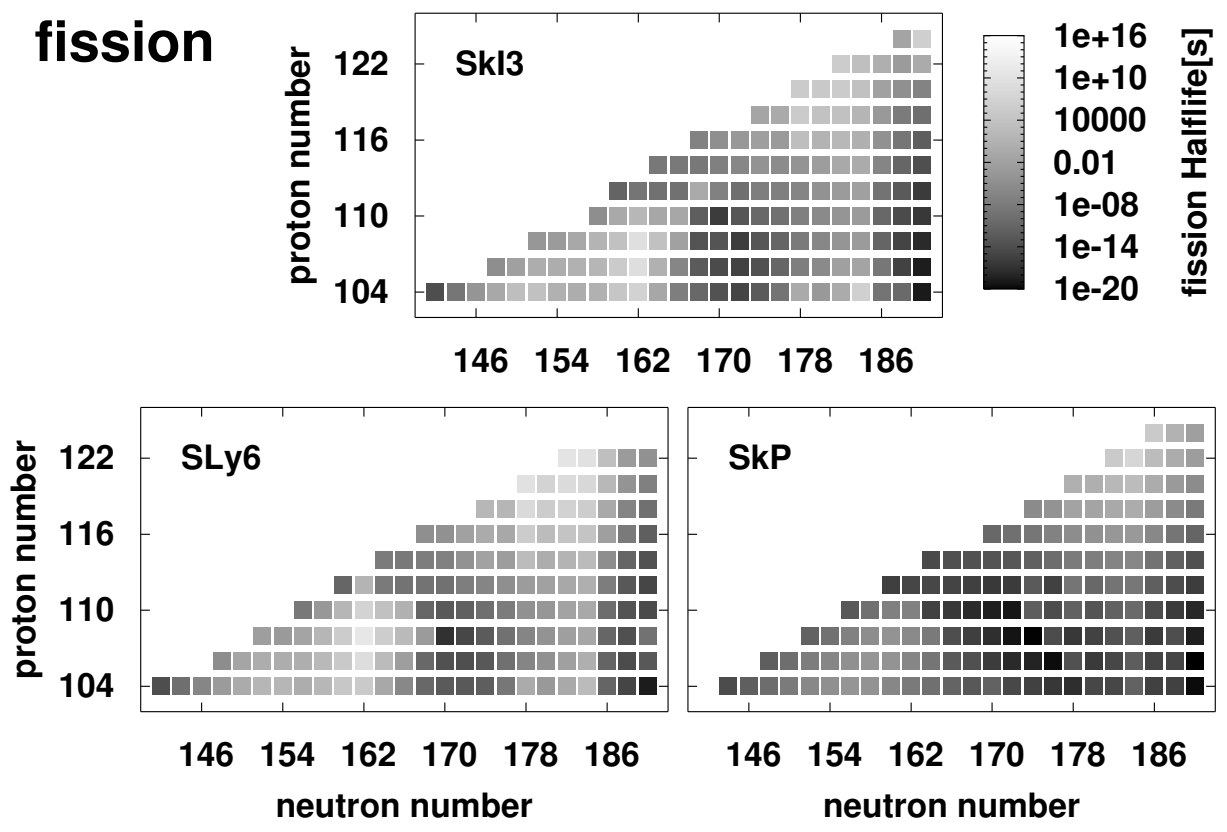

Fig. 4. Fission life-times for a broad variety of super-heavy elements and for three Skyrme parameterizations as indicated.

hope for a similarly simple direct connection between fission barrier and fission lifetime. A comparison of figure 3 with 4 shows that the very gross trends may look similar. We have checked whether one could establish a simple relation between barriers and lifetimes. This turned out to be impossible. Fission life-times are very subtle quantities which depend on more ingredients than just barriers alone.

There exist a few fission life-times along the decay channel from the upper island of stability. Fig. 6 compares computed life-times with data for fission and $\alpha$-decay. The $\alpha$ life-times are well reproduced by the calculations, but the fission life-times are dramatically underestimated towards the lower end of the chain. The reason for that is still unclear. The mismatch calls for further investigations.

\section{Conclusions}

Fission life-times have been computed using the Skyrme-Hartree-Fock method. The fission path has been generated with a quadrupole constraint where only axially symmetric deformations are considered. For the corresponding collective mass, we use self-consistent cranking. The quantum corrections to the collective potential (angular momentum projection, vibrational zero-point energy) are properly taken into account. The fission life-time is computed for thus given potential and mass by the WKB approximation, while the ground state energy, which is at the same time 


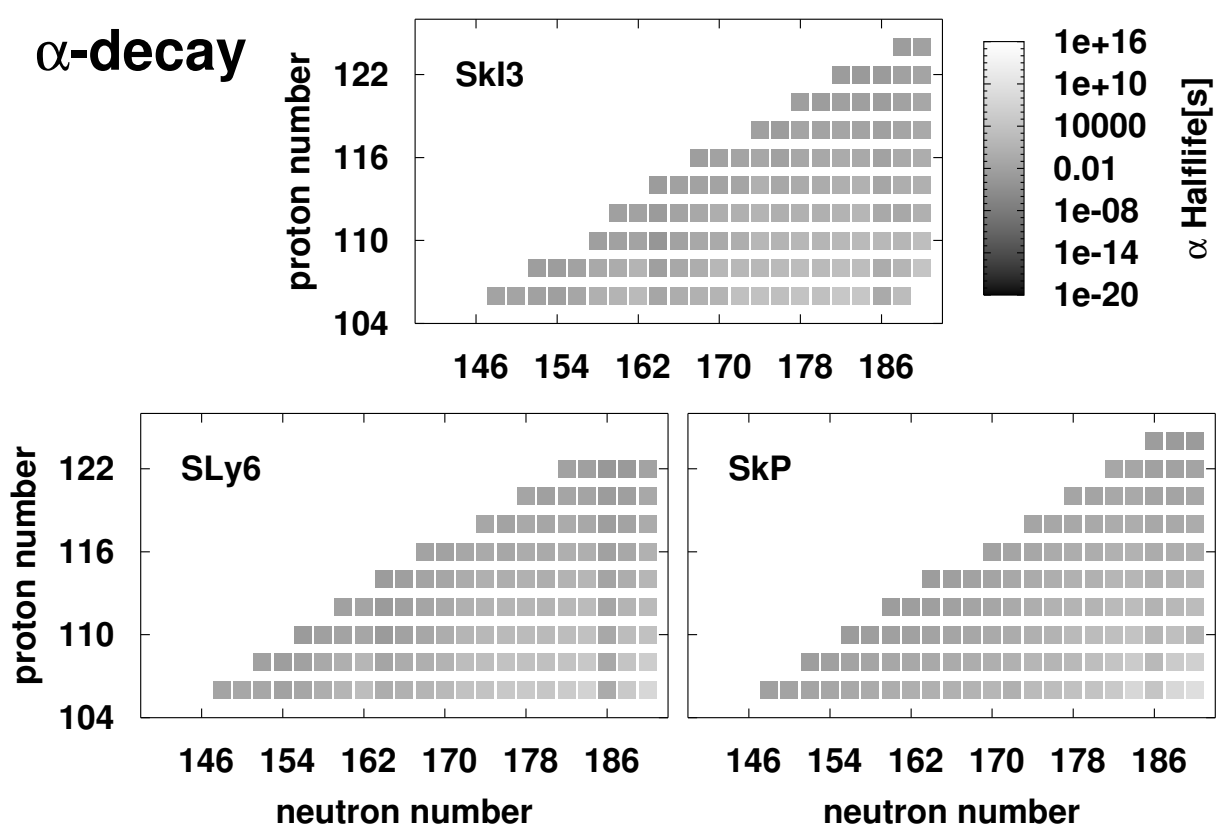

Fig. 5. $\alpha$-decay lifetime for a broad variety of super-heavy elements and for three Skyrme parameterizations as indicated.
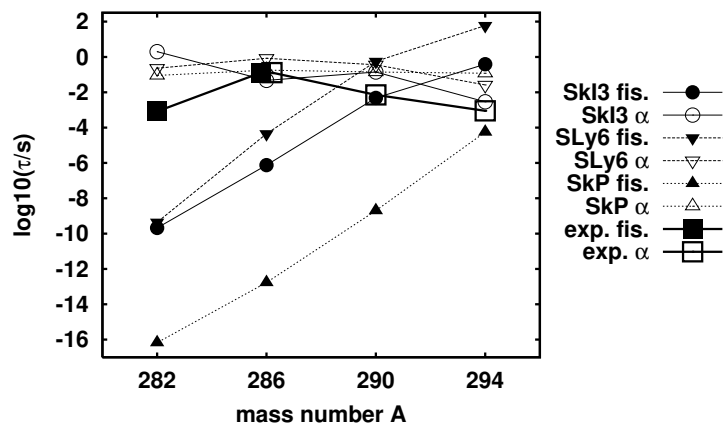

Fig. 6. Fission life-times and $\alpha$-decay life-times for elements along the decay chain from $Z / N=118 / 176$ computed with three different Skyrme parameterizations, as indicated, and compared with experimental data from 20 .

the entrance energy for fission, is computed quantum mechanically. Results have been produced for three different Skyrme forces, SkP, SLy6 and SkI3, to explore the sensitivity to the parameterization. For comparison, we have also computed the $\alpha$-decay life-times using the Viola systematics.

A first test was performed by comparing with known fission life-times in the lower region of super-heavy elements, $Z=104-108$. The theoretical results gather around the experimental values with deviations of \pm 2 orders of magnitude. That can be called a promising agreement, the more so, as the isotropic trends are well 
reproduced. The second test went for fission and $\alpha$-decay life-times in the $\alpha$-decay chain from 118/176. The $\alpha$-decay life-times are well reproduced. But it turned out that the fission life-times are grossly underestimated by all three forces towards the lower end of the chain at 112/170. The reasons for that mismatch are not yet clear. It is to be reminded that these super-heavy nuclei have extremely soft deformation energy surfaces with several shape isomers which complicates the determination of the ground state. Notwithstanding that open problem, we have produced a systematic survey of fission barriers, fission life-times and $\alpha$-decay life-times for all conceivable super-heavy elements in the range $\mathrm{Z}=104-124$ and $\mathrm{N}=150-190$. Barriers and fission life-times show nicely the islands of shell stabilization around 104/152 and 120/184. All forces predict a band of fission instability being orthogonal to the line connecting the two islands and crossing that around 112/166. There seems to be no path which could connect the 120/184 region with the lower island while avoiding fission. While the fission life-times show dramatic variation over the chart of super-heavy elements (from instability to $10^{16} \mathrm{~s}$ ), the $\alpha$-decay times vary gently with small overall changes and without visible shell effects. The general crossover from $\alpha$-decay to fission along the decay chains from the upper island is qualitatively reproduced by all three forces in the survey.

Altogether, the results are promising and challenging at the same time. They call for further investigations exploring more systematically the sensitivity to the Skyrme parameterization and improving the description in the very soft transitional region around 112/166.

\section{Acknowledgments}

We thank the regional computing center of the university Erlangen-Nürnberg for generous supply of computer time for the demanding calculations. The work was supported by the BMBF under contracts 06 ER 808 .

\section{References}

1. M. Bender, P.-H. Heenen, and P.-G. Reinhard, Rev. Mod. Rhys. 75, 121 (2003).

2. D. Vretenar, A.V. Afanasjev, G.A. Lalazissis, P. Ring, Phys. Rep., 409, 101 (2005).

3. J.R. Stone and P.-G. Reinhard, Prog. Part. Nucl. Phys. 58, 587 (2007).

4. J. Bartel, P. Quentin, M. Brack, C. Guet, and H.-B. Håakansson, Nucl. Phys. A386, 79 (1982).

5. J.-F. Berger, L. Bitaud, J. Decharge, M. Girod, and K. Dietrich, Nucl. Phys. A685, 1c (2001).

6. M. Warda et al, Phys. Scr. T, 125, 226 (2006).

7. P.-G. Reinhard and K. Goeke, Rep. Prog. Phys. 50, 1 (1987).

8. P. Möller, J. R. Nix, and W. J. Swiatecki, Nucl. Phys. A469, 1 (1987).

9. R. Smolańczuk, J. Skalski, and A. Sobiczewski, Phys. Rev. C 52, 1871 (1995).

10. J. Erler, P. Klüpfel, and P.-G. Reinhard, Eur. Phys. J. A, 37,81 (2008)

11. J. Dobaczewski, H. Flocard, and J. Treiner, Nucl. Phys. A422, 103 (1984).

12. P.-G. Reinhard and H. Flocard, Nucl. Phys. A584, 467 (1995). 
13. E. Chabanat, P. Bonche, P. Haensel, J. Meyer, and R. Schaeffer, Nucl. Phys. A627, 710 (1997).

14. P. Fleischer, P. Klüpfel, P.-G. Reinhard, and J. A. Maruhn, Phys. Rev. C, 70, 054321 (2004)

15. P. Klüpfel, J. Erler, P.-G. Reinhard, and J. A. Maruhn, Eur. Phys. J. A, 37,343 (2008)

16. J. Skalski, Phys. Rev. C 77, 064610 (2008).

17. T. Bürvenich et al, Phys. Rev. C, 69, 014307 (2004).

18. P. Möller et al, Nature 409, 785 (2001).

19. Yu. Ts. Oganessian et al, Eur. Phys. J. A, 15, 201 (2002).

20. Yu. Ts. Oganessian et al, Phys. Rev. C, 74, 044602 (2006).

21. G. Audi et al, Nucl. Phys. A, 729, 3 (2003).

22. F. P. Hessberger et al, Z. Phys. A, 359, 415 (1997).

23. J. M. Gates et al, Phys. Rev. C, 77, 034603 (2008).

24. K. Gregorich et al, Phys. Rev. C, 74, 044611 (2006).

25. J. Dvorak et al, Phys. Rev. Lett., 100, 132503 (2008).

26. S. Hofmann et al, Eur. Phys. J. A, 10, 5 (2001).

27. M. Bender, W. Nazarewicz, and P.-G. Reinhard, Phys. Lett. B , 515, 42 (2001).

28. C. Samanta, P. Roy Chowdhury, and D.N. Basu, Nucl. Phys. A 789, 142 (2007). 